\title{
MODELAGEM DO CRESCIMENTO E PRODUÇÃO EM CLASSES DE PRECIPITAÇÃO PLUVIOMÉTRICA PARA Eucalyptus sp.
}

\author{
Rodrigo Otávio Veiga de Miranda ${ }^{1}$, Andrea Nogueira Dias ${ }^{2}$, Afonso Figueiredo Filho ${ }^{2}$, \\ Izabele Domingues Soares ${ }^{1}$, Jovane Pereira da $\mathrm{Cruz}^{3}$ \\ ${ }^{1}$ Universidade Federal do Paraná, Programa de Pós-Graduação em Engenharia Florestal, Curitiba, Paraná, Brasil - \\ rov_miranda@yahoo.com.br; izabele.soares@gmail.com \\ ${ }^{2}$ Universidade Estadual do Centro-Oeste, Departamento de Engenharia Florestal, Irati, Paraná, Brasil - andias@irati.unicentro.br; \\ afonso.figueiredo@pq.cnpq.br \\ ${ }^{3}$ Bahia Specialty Cellulose, Alagoinhas, Bahia, Brasil - jovanecruz@gmail.com
}

Recebido para publicação: 02/10/2013 - Aceito para publicação: 06/06/2014

\begin{abstract}
Resumo
Uma das etapas fundamentais na modelagem do crescimento e produção é a identificação dos sítios, geralmente determinados pela relação entre altura dominante e idade. Porém há situações em que se torna interessante o uso de outras variáveis, como a precipitação pluviométrica, devido à grande correlação com a produtividade do povoamento. O objetivo deste estudo foi verificar a influência da estratificação por classes de precipitação pluviométrica no crescimento e produção volumétrica de Eucalyptus sp. Os dados provêm de plantios de híbridos de Eucalyptus grandis W. Hill ex Maiden x Eucalyptus urophylla S. T. Blake, do estado da Bahia, distribuídos em sete classes de precipitação, consideradas como estratos conforme o teste de Dette e Neumeyer (2001). Foram determinados os índices de sítio a cada classe de precipitação, sendo utilizada a área basal inicial média em cada um desses sítios. O modelo de Clutter foi empregado para a modelagem e as estimativas de produção foram comparadas pelo teste $\mathrm{F}$ de Graybill. A precipitação média influenciou no crescimento em altura dominante. A modelagem realizada por classe de precipitação gerou resultados similares àqueles obtidos pela modelagem sem estratificação, porém com maior precisão, sendo considerada adequada para estimar a produção para povoamentos clonais de eucalipto.
\end{abstract}

Palavras-chave: Eucalipto; ANCOVA não paramétrica; modelo de Clutter.

\begin{abstract}
Modeling of growth and yield by pluviometric precipitation classes for Eucalyptus sp. One of the key steps in modeling of growth and yield is the identification of different sites, which are generally determined by the relation of dominant height and age. However, there are situations in which it becomes interesting to use other variables, such as pluviometric precipitation, due to the high correlation with the productivity of the stand. This research aims to investigate influence of stratification by classes of precipitation in volumetric growth and yield of Eucalyptus sp. We collected data in hybrid stands of Eucalyptus grandis W. Hill ex Maiden x Eucalyptus urophylla S. T. Blake, from the state of Bahia, Brazil; such stands were distributed in seven classes of precipitation, each considered a site accordingly to Dette and Neumeyer (2001) test. We determined the site index for each class of precipitation, using the initial average basal area in each site. The Clutter model was used for modeling, and production estimates compared by Graybill $\mathrm{F}$ test. The average precipitation influenced the dominant height growth. The modeling performed by precipitation classes generated similar results to those obtained by modeling without stratification, but more accurately; we can consider it appropriate to estimate production to clonal eucalyptus stands.

Keywords: Eucalypt; nonparametric ANCOVA; Clutter model.
\end{abstract}

\section{INTRODUÇÃO}

Um modelo de crescimento e produção florestal é uma abstração da dinâmica de um povoamento florestal, que pode englobar o crescimento em sua totalidade ou das árvores que o constituem, a mortalidade e quaisquer outras alterações na composição de sua estrutura (FREIRE, 2002). Existe grande variedade de modelos de crescimento e produção na literatura, que variam desde simples equações que expressam a

FLORESTA, Curitiba, PR, v. 45, n. 1, p. 117 - 128, jan. / mar. 2015.

Miranda, R. O. V. de. et al.

ISSN eletrônico 1982-4688 / ISSN impresso 0015-3826

117

DOI: 10.5380/rf.v45i1.33664 
produção por unidade de área em função da idade, sítio e densidade do povoamento, até complexos sistemas de equações que simulam o crescimento individual das árvores de um povoamento, em função de suas próprias características e de árvores vizinhas e da distância entre elas (CLUTTER et al., 1983).

Os modelos de crescimento e produção podem ser classificados em modelos em nível de povoamento, por classe diamétrica e de árvores individuais (DAVIS; JOHNSON, 1987). Os modelos em nível de povoamento, às vezes denominados modelos globais, não explicam diretamente a variação do tamanho das árvores dentro do povoamento. No Brasil, representam os modelos de crescimento e produção florestal mais utilizados, os quais são baseados nas características do povoamento, como idade, área basal e índice de sítio. Essa modelagem tem sido realizada, basicamente, a partir do modelo de Clutter (1963), para diferentes espécies, clones e regiões, gerando estimativas precisas e livres de tendência (CRUZ, 2005; DIAS et al., 2005; CAMPOS; LEITE, 2013).

Uma das etapas fundamentais na modelagem do crescimento e produção florestal é a determinação da capacidade produtiva, que pode ser realizada por métodos diretos e indiretos. O método indireto é baseado em características das espécies, solo e condições climáticas da área. Já o método direto é realizado a partir de informações sobre a produtividade de espécies que já tenham sido introduzidas no local ou mesmo pelo crescimento médio da altura dominante em função da idade, podendo ser definido como um índice de sítio (SCOLFORO, 2006).

Além da altura dominante, há situações em que se torna vantajoso o uso de variáveis relacionadas à espécie, atributos do solo e características do clima, entre outras variáveis ambientais, na determinação das áreas com crescimento homogêneo, definidas por estratos (HUNTER; GIBSON, 1984; CORONA et al., 1998). Uma das variáveis ambientais importantes para a determinação desses estratos, em povoamentos de eucalipto, é a precipitação pluviométrica, devido à correlação positiva com o incremento em altura dominante, possuindo assim grande correlação com a produtividade do povoamento florestal (SOUZA et al., 2006; FEREIRA, 2009).

Maestri (2003) utilizou técnicas de estatística multivariada e análise de regressão múltipla, pelo procedimento Backward, para seleção das variáveis ambientais a serem correlacionadas com o crescimento em altura dominante em povoamentos clonais de híbridos de Eucalyptus grandis, situados na região Sudeste do Brasil. A precipitação pluviométrica, juntamente com a variação da evapotranspiração potencial e da temperatura máxima e mínima, foi umas das variáveis ambientais mais significativas na explicação do incremento anual em altura dominante.

Temps (2005) incorporou a variável precipitação média e suas transformações no modelo de Richards de incremento corrente em altura dominante em povoamentos de Pinus taeda L. não desbastados, com idades de 5 a 35 anos, em Santa Catarina. Isso propiciou ganhos em precisão nas estimativas da altura dominante e, consequentemente, em todo o sistema de prognose, reduzindo o erro padrão da estimativa, em média, em 7,5\%.

Souza et al. (2006) estudaram o efeito da precipitação pluviométrica no incremento periódico mensal em volume para povoamentos de eucalipto situados em regiões da bacia do rio Doce, Minas Gerais. Os autores verificaram correlação positiva acima de $80 \%$ entre a precipitação pluviométrica da estação chuvosa em cada ano com o ganho em volume.

Koehler (2009), trabalhando num povoamento jovem de Araucaria angustifolia (Bert.) Ktze., localizado nos municípios de Tijucas do Sul e Agudos do Sul, Paraná, verificou a correlação das propriedades físicas e químicas do solo com o incremento médio e corrente da espécie, usando o índice de correlação de Pearson. O autor detectou que essa correlação é mais acentuada para alguns elementos, em especial $\mathrm{N}$ e Al. Aspectos físicos também influenciaram no ritmo de crescimento, sobretudo a profundidade do solo.

Ferreira (2009) avaliou a correlação de variáveis meteorológicas e edáficas nos modelos de incremento, para povoamentos de eucalipto no extremo sul da Bahia. Foi observado que ocorreu correlação entre o incremento corrente anual em altura dominante, área basal e volume com as variáveis ambientais, especialmente precipitação mensal, déficit e excedente hídrico. Além disso, o índice de sítio obtido com a utilização de variáveis ambientais proporcionou ganho em precisão e melhor distribuição dos resíduos.

Diante do exposto, o objetivo desse trabalho foi verificar a influência da estratificação por classes de precipitação pluviométrica no crescimento e na produção volumétrica de um povoamento clonal de Eucalyptus sp., na região nordeste do estado da Bahia. 


\section{MATERIAL E MÉTODOS}

\section{Descrição da base de dados}

O trabalho foi realizado utilizando-se dados obtidos de uma rede de parcelas permanentes provenientes de um inventário florestal contínuo, estabelecida em plantios de híbridos de Eucalyptus grandis W. Hill ex Maiden x Eucalyptus urophylla S. T. Blake, com área útil por planta de $9 \mathrm{~m}^{2}$, localizados na região nordeste do estado da Bahia. Foram utilizadas 1.119 parcelas, com idades variando de 27 a 78 meses, medidas nos anos de 2007 a 2010. A intensidade de amostragem foi de, aproximadamente, uma parcela a cada 12,5 hectares.

As parcelas são circulares, com raio de 12,25 m (que representa uma área de 471,44 m²). Em cada parcela, foram medidos os diâmetros à altura do peito (DAP - 1,3 m do solo) com casca de todas as árvores da parcela, a altura total $(h)$ das 15 primeiras árvores e a altura total das cinco árvores dominantes $\left(h_{d o m}\right)$, segundo a metodologia proposta por Assmann (1961).

A precipitação adotada foi baseada nas isoietas anuais médias do estado da Bahia (COMPANHIA DE PESQUISA DE RECURSOS MINERAIS (CPRM), 2006), com a escala da base cartográfica digital de 1:1.000.000. Os dados abrangeram áreas com precipitação variando de 900 a $1.600 \mathrm{~mm}$, com amplitude de variação de $100 \mathrm{~mm}$, com exceção à classe de $1.500 \mathrm{~mm}$, por não possuir remedições, totalizando sete classes.

As estimativas de altura total, volume total da árvore e de altura dominante foram obtidas considerando a base de dados sem estratificação e também estratificada pelas classes de precipitação (PPT), ou seja, foram adotados os modelos de melhor desempenho a cada situação (MIRANDA, 2012), os quais podem ser observados a seguir. Os valores entre parênteses indicam o coeficiente de determinação ajustado e o erro padrão da estimativa percentual, respectivamente.

Dados sem estratificação em classes de PPT

$$
\begin{aligned}
& \operatorname{Ln}(h)=0,57254^{*}+0,86060^{*} \operatorname{Ln}\left(h_{d o m}\right)-0,31099 * \operatorname{Ln}\left(\frac{d g}{D A P}\right)+13,7971 *\left(\frac{1}{I D A P}\right)-3,27504 *\left(\frac{1}{D A P}\right)(0,95 ; 4,8 \%) \\
& V=-10,002 * D A P^{1,7770^{*}} h^{1,1210^{*}}(0,99 ; 8,4 \%) \\
& h_{d o m}=42,7924 * e^{\left(-1,4364^{*} 0,985 \mathrm{~F}^{I}\right)}(0,43 ; 15,4 \%)
\end{aligned}
$$

Dados estratificados em classes de PPT

$$
\begin{aligned}
& 900 \mathrm{~mm} \\
& \operatorname{Ln}(h)=0,6454 *+0,8375^{*} \operatorname{Ln}\left(h_{d o m}\right)-0,2747 * \operatorname{Ln}(d g / D A P)+5,2155^{*}(1 / I D A P)-3,0630 *(1 / D A P)(0,92 ; 4,5 \%) \\
& V=0,0042^{n s}+0,0001^{*} D A P^{2}+0,00002^{*} D A P^{2} h+0,00001^{*} D A P h^{2}-0,0001^{n s} h^{2}(0,99 ; 6,6 \%) \\
& h_{d o m}=1,9753^{*}\left[1+26,5288^{*} I^{1,3726 *}\right]^{0,2606}(0,36 ; 11,8 \%) \\
& 1.000 \mathrm{~mm} \\
& \operatorname{Ln}(h)=0,3894 *+0,9237 * \operatorname{Ln}\left(h_{d o m}\right)-0,2672 * \operatorname{Ln}(d g / D A P)+23,5110 *(1 / I D A P)-3,5298 *(1 / D A P)(0,93 ; 4,2 \%) \\
& V=0,004^{n s}+0,0045^{*} D A P-0,0004 * D A P^{2}+0,0004 * D A P h+0,00003^{*} D A P^{2} h-0,003 * h(0,99 ; 4,7 \%) \\
& h_{d o m}=29,8451^{*}\left[1-e^{(-0,0184 * I)}\right]^{0,6449}(0,54 ; 9,6 \%)
\end{aligned}
$$

\section{$1.100 \mathrm{~mm}$}

$\operatorname{Ln}(h)=0,1865^{*}+0,9748^{*} \operatorname{Ln}\left(h_{d o m}\right)-0,3706^{*} \operatorname{Ln}(d g / D A P)+36,5686^{*}(1 / I D A P)-3,3510 *(1 / D A P)(0,93 ; 5,1 \%)$

$V=-9,9479 * D A P^{1,8164 *} h^{1,0707}(0,99 ; 8,6 \%)$

$h_{\text {dom }}=29,0068 *\left[1-e^{(-0,0263 * I)}\right]^{0,9221}(0,48 ; 11,9 \%)$

$1.200 \mathrm{~mm}$

$\operatorname{Ln}(h)=0,7497 *+0,8431 * \operatorname{Ln}\left(h_{\text {dom }}\right)-0,2064 * \operatorname{Ln}(d g / D A P)+36,2265 *(1 / I$ DAP $)-5,5905 *(1 / D A P)(0,91 ; 4,8 \%)$ 


$$
\begin{aligned}
& V=0,0016^{n s}+0,0001^{*} D A P^{2}+0,00002^{*} D A P^{2} h+0,00001^{*} D A P h^{2}-0,00002^{n s} h^{2}(0,99 ; 10,1 \%) \\
& h_{d o m}=37,2201^{*}\left[1-e^{\left(-0,0113^{*} I\right)}\right]^{0,5584}(0,54 ; 9,7 \%) \\
& 1.300 \mathrm{~mm} \\
& L n(h)=0,3774^{*}+0,9287^{*} \operatorname{Ln}\left(h_{d o m}\right)-0,2628^{*} \operatorname{Ln}(d g / D A P)+38,9009^{*}(1 / I D A P)-4,0185^{*}(1 / D A P)(0,94 ; 4,5 \%) \\
& V=-9,9147 * D A P^{1,8256^{*}} h^{1,0495}(0,99 ; 9,2 \%) \\
& h_{d o m}=28,5244 *\left[1-e^{(-0,0325 * I)}\right]^{1,0599}(0,55 ; 11,2 \%) \\
& 1.400 \mathrm{~mm} \\
& \operatorname{Ln}(h)=0,7083^{*}+0,8728^{*} \operatorname{Ln}\left(h_{d o m}\right)-0,1239 * \operatorname{Ln}(d g / D A P)+62,4286^{*}(1 / I D A P)-7,1228^{*}(1 / D A P)(0,95 ; 4,4 \%) \\
& V=-9,9291^{*} D A P^{1,8569 *} h^{1,0311}(0,99 ; 6,0 \%) \\
& h_{d o m}=9,1957^{*}+37,6828^{*} e^{\left(-44,1551^{-1,0195}\right)}(0,73 ; 9,1 \%) \\
& 1.600 \mathrm{~mm} \\
& \operatorname{Ln}(h)=0,6771^{*}+0,8550^{*} \operatorname{Ln}\left(h_{d o m}\right)-0,2743^{*} \operatorname{Ln}(d g / D A P)+30,1734 *(1 / I D A P)-5,0965^{*}(1 / D A P)(0,94 ; 5,3 \%) \\
& V=-10,0315^{*} D A P^{1,7684 *} h^{1,1392}(0,99 ; 6,4 \%) \\
& h_{d o m}=3,7269^{*} e^{\left(\frac{-22,9567}{I}\right)}(0,99 ; 10,9 \%)
\end{aligned}
$$

\section{Validação das classes de precipitação}

As estimativas de altura dominante geradas para cada uma das classes de PPT foram comparadas a partir do método proposto por Dette e Neumeyer (2001) $\left(\mathrm{T}_{\mathrm{N}}\right)$, no qual a estatística testa a igualdade de $k$ curvas de regressão, baseada numa estatística semelhante à análise de variância (ANOVA), mas inserido no contexto da estatística não paramétrica. Esse teste foi importante para verificar a necessidade de se ajustar um modelo de crescimento e produção para cada classe de PPT.

O procedimento é similar à análise de covariância baseada no método de Snedecor, descrita em Schneider et al. (2009), o qual testa a hipótese de não diferença de inclinação (coeficiente angular) e nos níveis (intercepto). Porém essa análise de covariância apresenta a restrição de ser aplicada apenas para modelos lineares simples.

A hipótese $H_{0}$ do teste $\mathrm{T}_{\mathrm{N}}$ representa a igualdade das curvas de regressão, ou seja, $f(1)=f(2)=\ldots$ $=f(k)$, em que $k$ representa o número de grupos ou curvas de regressão. A estatística do teste foi avaliada observando-se sua probabilidade de ser ou não significativa, a partir do $p$-valor $(p \leq 0,05)$. Para isso, o procedimento utiliza o processo de bootstrap, o qual realiza reamostragens a partir da base de dados, sendo que cada reamostragem gera um intervalo de confiança para a variável de interesse. Partindo-se do pressuposto de que a amostra é representativa da população-foco e considerando um número de reamostragens suficiente, torna-se possível gerar a curva de valores críticos para a variável de interesse. Essa estatística foi obtida com o uso do software R (R DEVELOPMENT CORE TEAM, 2012), versão 2.13.1, empregando-se o pacote fANCOVA (WANG, 2010).

\section{Modelagem do crescimento e da produção}

Foram construídas as curvas de índices de sítio a partir dos modelos descritos anteriormente empregando-se o método da curva-guia e considerando uma idade índice de 60 meses, a partir do modelo mais adequado em cada classe de PPT. As tabelas de limites de altura dominante geradas, tanto para os dados sem estratificação quanto para aqueles estratificados por classes de PPT, foram agrupadas em três classes de sítios (I, II e III).

Para realizar a modelagem do crescimento e da produção, os dados de volume $\left(\mathrm{m}^{3}\right.$.parcela.ha $\left.{ }^{-1}\right)$ e área basal $\left(\mathrm{m}^{2}\right.$.parcela.ha $\left.{ }^{-1}\right)$, assim como as estimativas de altura dominante (m.parcela $\left.{ }^{-1}\right)$, foram agrupados conforme a definição das classes de índices de sítio (dados sem estratificação) e das classes de índices de sítio construídas para cada uma das classes de PPT (dados estratificados). O modelo de crescimento e produção empregado foi desenvolvido por Clutter (1963), tendo como características 
principais ser do tipo global, de densidade variável, explícito e com características de compatibilidade e consistência. O modelo de Clutter está descrito a seguir (CLUTTER et al., 1983).

$$
\begin{gathered}
\operatorname{Ln}\left(V_{2}\right)=\beta_{0}+\beta_{1}\left(\frac{1}{I_{2}}\right)+\beta_{2} S_{1}+\beta_{3} \operatorname{Ln}\left(G_{2}\right)+\operatorname{Ln}\left(\varepsilon_{i}\right) \\
\operatorname{Ln}\left(G_{2}\right)=\operatorname{Ln}\left(G_{1}\right)\left(\frac{I_{1}}{I_{2}}\right)+\alpha_{0}\left(1-\frac{I_{1}}{I_{2}}\right)+\alpha_{1}\left(1-\frac{I_{1}}{I_{2}}\right) S_{1}+\operatorname{Ln}\left(\varepsilon_{i}\right)
\end{gathered}
$$

em que: $V_{2}=$ volume da i-ésima unidade de manejo, em $\mathrm{m}^{3} \cdot \mathrm{ha}^{-1}$, na idade $I_{2}$;

$G_{l}$ e $G_{2}=$ área basal da i-ésima unidade de manejo, em m².ha ${ }^{-1}$, na idade $I_{l}$ e $I_{2}$, respectivamente;

$I_{1}$ e $I_{2}=$ idade atual e futura do povoamento, em meses, respectivamente;

$S_{l}=$ índice de sítio da i-ésima unidade de manejo na idade $I_{l}$;

$L n=\log$ aritmo natural;

$\beta_{i}, \alpha_{i}=$ coeficientes a serem estimados;

$\varepsilon_{\mathrm{i}}=$ erro aleatório.

O sistema foi ajustado utilizando-se o software econométrico Gretl (COTTRELL; LUCCHETTI, 2013) versão 1.9.12, empregando-se o método dos mínimos quadrados em dois estágios. As estatísticas utilizadas para avaliar o ajuste foram o coeficiente de determinação ajustado $\left(R_{\text {ajust }}^{2}\right)$, o erro padrão da estimativa relativo $\left(S_{y x} \%\right)$ e a análise gráfica da distribuição de resíduos (DRAPER; SMITH, 1981). O nível de significância adotado nas análises foi $\alpha \leq 0,05$. Antes de gerar as estimativas a partir do modelo de Clutter, foi necessário estimar a área basal inicial $\left(G_{i}\right)$ em cada sítio considerado. Essa estimativa é muito importante, já que a escolha de um valor inadequado pode causar contradições nos valores estimados de volume nas tabelas de produção de densidade variável (TPDV), com a capacidade produtiva do local (CAMPOS et al., 1996). Geralmente, obtém-se a área basal média na idade inicial utilizando-a no sítio médio e, para os demais, utilizando essa média mais ou menos um ou dois desvios padrões, para o sítio mais e menos produtivo, respectivamente $\left(\bar{G}_{i} \pm \sigma_{G_{i}}\right.$ ou $\left.\bar{G}_{i} \pm \sigma_{2 G_{i}}\right)$.

Em seguida, foram construídas as tabelas de produção, assim como as curvas de incremento médio mensal (IMM) e incremento corrente mensal (ICM). A idade técnica de corte (ITC) foi determinada ao se igualarem as expressões de IMM e ICM. A partir dos resultados, foi observada a tendência das estimativas geradas e o comportamento da ITC com o aumento da capacidade produtiva.

As estimativas de produção das duas situações (sem estratificação e estratificada por classes de PPT) foram comparadas com os respectivos valores observados, a fim de verificar possíveis diferenças estatísticas. A comparação se deu a partir do teste F de Graybill (1976), adotando-se um nível de significância de 5\%, representando um teste de identidade de fácil aplicação e interpretação. Com o intuito de se verificar a precisão das estimativas obtidas pelas duas alternativas, foi calculado o erro padrão da estimativa relativo $\left(S_{y x} \%\right)$ para cada uma.

\section{RESULTADOS E DISCUSSÃO}

Na figura 1 são apresentadas as curvas de crescimento médio em altura dominante para as diferentes classes de precipitação. Ocorre uma tendência já esperada: nas regiões em que a precipitação foi maior, o crescimento médio em altura dominante foi superior. A curva referente à classe de $900 \mathrm{~mm}$ demonstra uma média de altura dominante inferior em todas as idades. Por outro lado, as curvas das classes de 1.400 e $1.600 \mathrm{~mm}$ apresentam a maior taxa de crescimento para essa variável. É importante ressaltar a proximidade entre essas curvas, indicando grande semelhança na tendência do incremento nesses locais. Devido a essas diferenças de crescimento em altura dominante, o que resulta em regiões de capacidade produtiva distintas, foi possível a identificação dos diferentes estratos.

\section{Validação das curvas de precipitação}

Foi aplicado o teste de Dette e Neumeyer (2001) para comparar as curvas de regressão. Primeiramente, o teste foi utilizado na comparação das curvas das classes de 900 a $1.600 \mathrm{~mm}$, sendo o resultado da estatística $\mathrm{T}_{\mathrm{N}}$ de 5,07* ( $p$-valor $\left.<0,05\right)$, indicando que pelo menos uma das curvas difere das 
demais. Com isso, foi realizada a comparação das curvas duas a duas, sendo os resultados apresentados na tabela 1 .

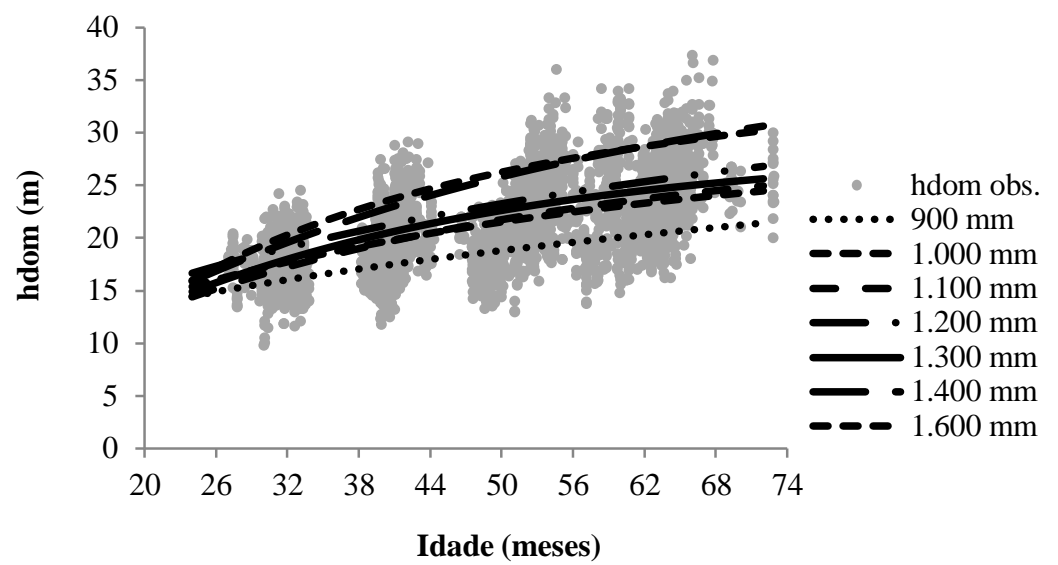

Figura 1. Crescimento médio em altura dominante para as diferentes classes de precipitação pluviométrica.

Figure 1. Average growth in dominant height for the different pluviometric precipitation classes.

Tabela 1. Teste de Dette e Neumeyer (2001) na comparação das curvas de crescimento médio em altura dominante, para diferentes classes de precipitação pluviométrica $(\mathrm{mm})$.

Table 1. Dette and Neumeyer (2001) test in the comparison of average dominant height growth curves, for different pluviometric precipitation classes $(\mathrm{mm})$.

\begin{tabular}{lccccc}
\hline Classes de PPT & TN & Classes de PPT & TN & Classes de PPT & TN \\
\hline 900 e 1.000 & $1,56^{*}$ & $1.000 \mathrm{e} 1.200$ & $0,77^{*}$ & $1.100 \mathrm{e} 1.600$ & $4,48^{*}$ \\
$900 \mathrm{e} 1.100$ & $1,76^{*}$ & $1.000 \mathrm{e} 1.300$ & $0,22^{*}$ & $1.200 \mathrm{e} 1.300$ & $0,24^{*}$ \\
$900 \mathrm{e} 1.200$ & $4,50^{*}$ & $1.000 \mathrm{e} 1.400$ & $4,31^{*}$ & $1.200 \mathrm{e} 1.400$ & $1,54^{*}$ \\
$900 \mathrm{e} 1.300$ & $2,89^{*}$ & $1.000 \mathrm{e} 1.600$ & $4,77^{*}$ & $1.200 \mathrm{e} 1.600$ & $1,77^{*}$ \\
$900 \mathrm{e} 1.400$ & $10,98^{*}$ & $1.100 \mathrm{e} 1.200$ & $0,74^{*}$ & $1.300 \mathrm{e} 1.400$ & $2,63^{*}$ \\
$900 \mathrm{e} 1.600$ & $11,76^{*}$ & $1.100 \mathrm{e} 1.300$ & $0,15^{*}$ & $1.300 \mathrm{e} 1.600$ & $3,01^{*}$ \\
$1.000 \mathrm{e} 1.100$ & $0,05^{*}$ & $1.100 \mathrm{e} 1.400$ & $3,97^{*}$ & $1.400 \mathrm{e} 1.600$ & $0,06^{*}$ \\
\hline
\end{tabular}

*Significativo considerando um nível de 5\% de significância.

Observa-se que, para as curvas de regiões cuja média em precipitação não difere muito, o valor da estatística $T_{N}$ foi menor em relação às demais. Mesmo curvas com padrões de crescimento bastante semelhantes, como as de 1.400 e $1.600 \mathrm{~mm}$, foram consideradas distintas, o que demonstra o rigor desse teste. Conforme os resultados, há evidência estatística para rejeitar a hipótese de nulidade, ou seja, estatisticamente não se observa igualdade das curvas de regressão comparadas. Assim, cada classe de PPT foi considerada como um estrato.

Dessa forma, foram obtidas as tabelas de limites de altura dominante para cada classe de PPT, agrupadas em três classes de sítio, assim como para os dados sem estratificação. Na tabela 2 estão apresentados os limites das classes de sítios e os respectivos intervalos de altura dominante na idade índice. Os limites estão dispostos em relação às classes de sítios I, II e III, correspondendo, respectivamente, a alta, média e baixa produtividade.

\section{Modelagem do crescimento e da produção}

Com a determinação do sítio, para cada situação foi realizada a modelagem do crescimento e da produção. Posteriormente, foi determinada a área basal nas idades iniciais. Neste trabalho não foi possível utilizar uma única média e os desvios, pois há idades em que algumas classes de sítio não foram representadas, o que poderia gerar subestimativas ou superestimativas em determinados sítios. Dessa forma, foi obtida uma média de área basal inicial em cada classe de sítio e em cada classe de PPT, de acordo com a estratificação. 
Tabela 2. Limites de altura dominante para as classes de sítio utilizadas na modelagem do crescimento e produção, na idade índice de 60 meses.

Table 2. Dominant height limits for site index classes used in the modeling of growth and yield, in the index age of 60 months.

\begin{tabular}{|c|c|c|c|c|c|c|}
\hline \multirow{3}{*}{$\begin{array}{l}\text { Classe de PPT (mm) } \\
\text { S/est. }\end{array}$} & \multicolumn{6}{|c|}{ Classes de sítio } \\
\hline & \multicolumn{2}{|c|}{$\mathbf{I}$} & \multicolumn{2}{|c|}{ II } & \multicolumn{2}{|c|}{ III } \\
\hline & 32,5 & 38,5 & 23,5 & 29,5 & 14,5 & 20,5 \\
\hline 900 & 27,5 & 30,5 & 21,5 & 24,5 & 15,5 & 18,5 \\
\hline 1.000 & 30,5 & 33,5 & 24,5 & 27,5 & 18,5 & 21,5 \\
\hline 1.100 & 28,8 & 32,2 & 22,0 & 25,4 & 15,2 & 18,6 \\
\hline 1.200 & 31,0 & 34,0 & 25,0 & 28,0 & 19,0 & 22,0 \\
\hline 1.300 & 30,5 & 33,5 & 24,5 & 27,5 & 18,5 & 21,5 \\
\hline 1.400 & 33,5 & 36,5 & 27,5 & 30,5 & 21,5 & 24,5 \\
\hline 1.600 & 35,8 & 39,3 & 28,8 & 32,3 & 21,8 & 25,3 \\
\hline
\end{tabular}

PPT: precipitação pluviométrica; S/est.: ajuste sem estratificação; I, II e III: classes de sítio de alta, média e baixa produtividade, respectivamente, considerando uma idade índice de 60 meses.

Dias (2000), trabalhando com dados de clones de híbridos de E. urophylla e E. grandis, empregou a média da área basal inicial aos 27 meses para ajustar o modelo de Clutter a plantios submetidos a desbaste, obtendo resultados satisfatórios na prognose da produção volumétrica do povoamento. Novaes (2009) empregou a média da área basal inicial observada aos 48 meses para modelar o crescimento e produção de um povoamento de teca, a partir de dados de análise de tronco, empregandose o modelo de Buckman e de Clutter, e obteve estimativas precisas da produção para este último. Nascimento (2010) utilizou a área basal inicial média aos cinco anos, área basal inicial média menos um desvio padrão e área basal inicial média mais um desvio padrão, para os sítios de média, baixa e alta produtividade, para a projeção volumétrica de um povoamento de pínus na região do Planalto Norte de Santa Catarina, conseguindo estimativas coerentes do ponto de vista biológico. Os coeficientes e estatísticas de ajuste e precisão do modelo de Clutter estão apresentados na tabela 3.

Tabela 3. Estimativa dos parâmetros do modelo de Clutter e as respectivas estatísticas de avaliação, em relação aos dados sem estratificação e estratificados em classes de PPT para Eucalyptus sp., na região nordeste da Bahia.

Table 3. Estimated parameters of the Clutter model and their statistical evaluation, related to the data without stratification and stratified by precipitation classes for Eucalyptus sp., in the northeastern of Bahia, Brazil.

\begin{tabular}{|c|c|c|c|c|c|c|c|c|c|c|}
\hline & \multicolumn{6}{|c|}{ Coeficientes } & \multicolumn{2}{|c|}{$V\left(m^{3} \cdot h^{-1}\right)$} & \multicolumn{2}{|c|}{$G\left(m^{2} \cdot h a^{-1}\right)$} \\
\hline & $\beta_{0}$ & $\beta_{1}$ & $\boldsymbol{\beta}_{2}$ & $\boldsymbol{\beta}_{3}$ & $\boldsymbol{\alpha}_{0}$ & $\alpha_{1}$ & $\boldsymbol{R}_{\text {ajust }}^{2}$ & $S_{y x} \%$ & $\boldsymbol{R}_{\text {ajust }}^{2}$ & $S_{y x} \%$ \\
\hline S/est. & $1,3694 *$ & $-21,8659 *$ & $0,0348 *$ & $1,2414 *$ & $1,207^{*}$ & $0,7432 * *$ & 0,9769 & 6,67 & 0,9373 & 6,57 \\
\hline 900 & $1,1282 *$ & $-28,7477 *$ & $0,0513 *$ & $1,1556^{*}$ & $2,5724 *$ & $0,032 *$ & 0,9521 & 6,09 & 0,8969 & 5,73 \\
\hline 1.000 & $1,6884 *$ & $-23,748 *$ & $0,0364 *$ & $1,1198 *$ & $2,9534 *$ & $0,1531 * *$ & 0,9409 & 5,68 & 0,9289 & 4,13 \\
\hline 1.100 & $1,3273 *$ & $-24,3588 *$ & $0,0396 *$ & $1,218^{*}$ & $1,7001 *$ & $0,5412 * *$ & 0,9558 & 6,08 & 0,9497 & 4,15 \\
\hline 1.200 & $1,1616^{*}$ & $-27,1194 *$ & $0,0302 *$ & $1,4457 *$ & $1,3973^{*}$ & $0,613 * *$ & 0,9351 & 6,01 & 0,8486 & 5,86 \\
\hline 1.300 & $1,104^{*}$ & $-21,3677^{*}$ & $0,034 *$ & $1,3713^{*}$ & $2,7798 *$ & $0,0949 * * *$ & 0,9346 & 6,64 & 0,8535 & 6,23 \\
\hline 1.400 & $2,2395^{*}$ & $-29,7109 *$ & $0,0328^{*}$ & $0,9447 *$ & $3,2755^{*}$ & $0,0118^{*}$ & 0,9564 & 5,43 & 0,9064 & 5,06 \\
\hline 1.600 & $1,6591^{*}$ & $-20,7033^{*}$ & $0,0327^{*}$ & $1,0835^{*}$ & $3,3881^{*}$ & $0,0767 * *$ & 0,9633 & 6,00 & 0,9341 & 4,93 \\
\hline
\end{tabular}

S/est.: ajuste sem estratificação; * coeficiente significativo a 5\% de significância; ** variável associada ao coeficiente foi modificada de $\left(1-I_{1} / I_{2}\right) S$ para $\left(1-I_{1} / I_{2}\right) \operatorname{Ln}\left(G_{1}\right)$; *** variável associada a coeficiente foi modificada de $\left(1-I_{1} / I_{2}\right) S$ para $\left(1-I_{1} / I_{2}\right) \operatorname{Ln}\left(G_{1}^{2}\right)$.

O coeficiente $\beta_{1}$ foi negativo para todos os ajustes, contribuindo na obtenção de estimativas consistentes. O coeficiente $\alpha_{1}$ teve o termo a ele associado $\left(1-I_{1} / I_{2}\right) S$ alterado para $\left(1-I_{1} / I_{2}\right) \operatorname{Ln}\left(G_{1}\right)$ no ajuste sem estratificação e para as classes $1.000,1.100,1.200$ e $1.600 \mathrm{~mm}$; e para $\left(1-I_{1} / I_{2}\right) \operatorname{Ln}\left(G_{1}^{2}\right)$ na classe de $1.300 \mathrm{~mm}$, pois na forma original forneceram estimativas de produção incoerentes do ponto de vista biológico. Considerando-se o ajuste sem estratificação, os valores de $R_{a j u s t}^{2}$ e $S_{y x} \%$, tanto para 
volume quanto para área basal, apresentaram-se superiores aos encontrados por Dias (2000), Dias et al. (2005) e Oliveira (2012).

Em relação aos ajustes estratificados por classes de PPT, o $R_{\text {ajust }}^{2}$ variou de 0,9346 a 0,9633 , e o $S_{y x} \%$ oscilou de 5,43 a 6,64\% para as estimativas de produção. Para estimativas de área basal, a variação foi de 0,8486 a 0,9497 para o $R_{a j u s t}^{2}$ e de 4,13 a $6,23 \%$ para o $S_{y x} \%$, sendo ambos considerados adequados para todas as classes, com valores próximos àqueles obtidos com o ajuste sem a estratificação em classes de PPT.

$\mathrm{Na}$ figura 2 tem-se a dispersão de resíduos para os ajustes do modelo de Clutter, sem estratificação e estratificados por classe de PPT. As estimativas se apresentaram livres de tendências, tanto para a produção volumétrica (a) quanto para as estimativas de área basal (b). Para o ajuste estratificado por classe de PPT, as estimativas de produção e área basal também apresentaram distribuições de resíduos não tendenciosas.

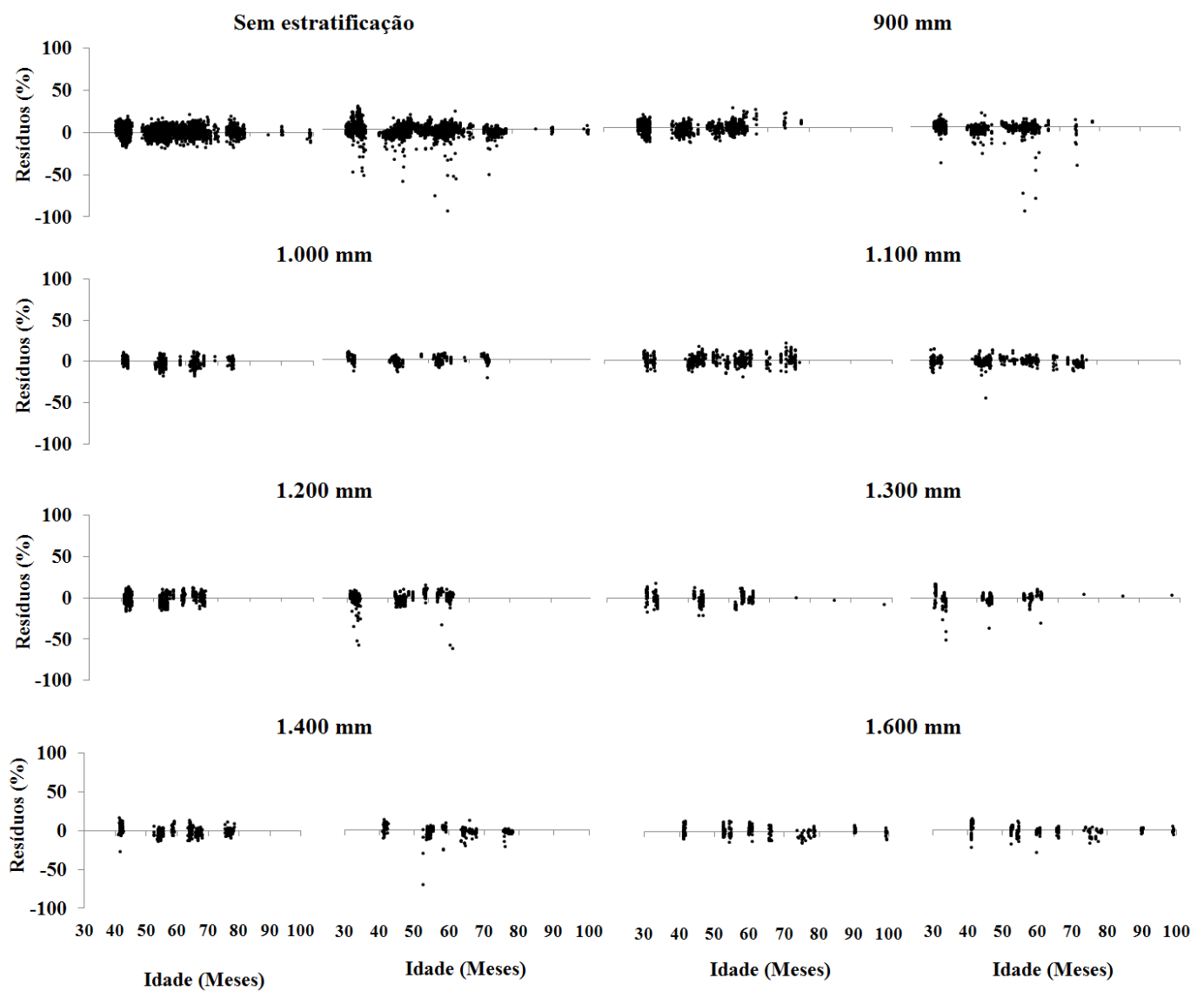

(a)

(b)

(a)

(b)

Figura 2. Distribuição de resíduos para a equação de produção volumétrica (a) e área basal (b) do modelo de Clutter, relativa aos dados sem estratificação e estratificados em classes de PPT para Eucalyptus sp., na região nordeste da Bahia.

Figure 2. Residual distribution for the volumetric yield equation (a) and basal area equation (b) of the Clutter model related to data with and without stratification by precipitation classes for Eucalyptus sp., in the northeastern of Bahia, Brazil.

O modelo de Clutter foi empregado para a obtenção das estimativas de produção, considerandose o ajuste sem estratificação e o estratificado por classes de PPT. Na tabela 4 estão apresentadas as informações de área basal nas idades iniciais e na idade de máximo incremento médio, em cada um dos sítios, as produções e as respectivas idades técnicas de corte, sintetizadas de modo a facilitar a 
visualização e comparação das diferentes classes de sítio e precipitação. As produções e idades técnicas de corte seguiram uma tendência já esperada do ponto de vista biológico: sítios mais produtivos atingiram a idade de máximo incremento médio mais cedo. Além disso, na medida em que se aumentou o valor de índice de sítio, maiores valores de produção da área basal foram encontrados.

As produções estimadas sem e com estratificação em classes de PPT foram comparadas pelo teste $\mathrm{F}$ de Graybill. De acordo com o teste, tanto as estimativas de produção da área sem estratificação quanto aquelas para a área estratificada pelas classes de PPT não diferiram estatisticamente dos valores observados de produção, obtendo-se valores de F de 0,79 e 2,64, respectivamente, admitindo-se um valor de F tabelado de 3,00. Resultado similar foi obtido por Soares et al. (2007), que compararam volumes prognosticados com os valores observados, por classe diamétrica, para dados de eucalipto híbrido estaca (E. grandis e E. urophylla) de primeira rotação. Na ocasião, utilizaram o teste $\mathrm{L} \& \mathrm{O}$ e encontraram valor inferior a um para o F de Graybill.

Pode-se inferir que as estimativas geradas a partir do modelo global nas duas condições de ajuste (não estratificado e estratificado por classes de PPT) não diferiram estatisticamente dos valores observados por ocasião das medições, sendo consideradas adequadas para estimar a produção para povoamentos clonais de eucalipto. Dessa maneira, o teste indica que a geração de modelos de produção inserindo classes de precipitação gerou resultados similares àqueles obtidos pelo método de modelagem sem estratificação.

Tabela 4. Idade técnica de corte e produção $(\mathrm{G}$ e $\mathrm{V})$ por hectare, para o ajuste sem estratificação e estratificado em classes de PPT para Eucalyptus sp., na região nordeste da Bahia.

Table 4. Technique age for cutting and yield ( $\mathrm{G}$ and $\mathrm{V}$ ) by hectare, for adjustment without stratification and stratified by precipitation classes for Eucalyptus sp., in the northeastern of Bahia, Brazil.

\begin{tabular}{lccccccccccccc}
\hline Classe PPT & $\mathbf{S}$ & $\mathbf{G}_{\mathbf{i}}$ & $\mathbf{I}$ & $\mathbf{G}$ & $\mathbf{V}$ & $\mathbf{I T C}$ & Classe PPT & $\mathbf{S}$ & $\mathbf{G}_{\mathbf{i}}$ & $\mathbf{I}$ & $\mathbf{G}$ & $\mathbf{V}$ & $\mathbf{I T C}$ \\
\hline \multirow{3}{*}{ S/est. } & 34 & 15,0 & 31 & 18,8 & 316,2 & 50 & & 32,5 & 13,9 & 33 & 16,2 & 279,7 & 51 \\
& 25 & 12,7 & 28 & 17,6 & 218,0 & 53 & 1.200 & 26,5 & 12,6 & 30 & 15,5 & 224,6 & 53 \\
& 16 & 8,9 & 28 & 14,2 & 130,2 & 63 & & 20,5 & 12,4 & 30 & 15,5 & 187,9 & 54 \\
\hline \multirow{3}{*}{900} & 29 & 14,0 & 29 & 21,6 & 290,1 & 58 & & 32 & 13,4 & 29 & 18,3 & 320,9 & 52 \\
& 23 & 10,6 & 28 & 17,6 & 170,9 & 60 & 1.300 & 26 & 12,2 & 30 & 17,8 & 260,4 & 57 \\
& 17 & 8,5 & 28 & 14,4 & 100,8 & 61 & & 20 & 10,6 & 30 & 17,2 & 210,0 & 63 \\
\hline \multirow{3}{*}{1.000} & 32 & 16,9 & 41 & 18,9 & 293,3 & 51 & & 35 & 20,6 & 41 & 24,6 & 357,9 & 56 \\
& 26 & 10,7 & 30 & 17,5 & 232,0 & 60 & 1.400 & 29 & 13,4 & 30 & 22,2 & 274,2 & 59 \\
& 20 & 9,1 & 30 & 16,8 & 183,0 & 65 & & 23 & 12,0 & 30 & 20,3 & 210,9 & 61 \\
\hline \multirow{3}{*}{1.100} & 30,5 & 13,0 & 38 & 15,4 & 224,1 & 54 & & 37,5 & 13,7 & 27 & 21,8 & 336,8 & 51 \\
& 23,7 & 10,5 & 30 & 14,8 & 167,5 & 57 & 1.600 & 30,5 & 12,8 & 27 & 21,5 & 267,0 & 53 \\
& 16,9 & 8,2 & 30 & 13,3 & 118,6 & 66 & & 23,5 & 14,4 & 39 & 20,4 & 212,7 & 62 \\
\hline
\end{tabular}

S/est.: ajuste sem estratificação; V: produção em volume $\left(\mathrm{m}^{3} \cdot \mathrm{ha}^{-1}\right)$; I: idade, em meses; $\mathrm{S}$ : classe de sítio; $\mathrm{G}_{\mathrm{i}}$ : área basal inicial $\left(\mathrm{m}^{2} \cdot \mathrm{ha}^{-1}\right)$; ITC: idade técnica de corte (meses).

Embora, estatisticamente, não tenham ocorrido diferenças entre os valores observados de volume por unidade de área com os valores estimados a partir dos dados sem estratificação e os dados estratificados por classes de PPT, tornou-se importante identificar quais estimativas foram mais precisas. Para isso, foi calculado o erro padrão da estimativa relativo $\left(S_{y x} \%\right)$. Para o ajuste sem estratificação, esse erro foi de 6,67\% na estimativa de produção. Santana et al. (2005), trabalhando com dados de plantios seminais de Eucalyptus grandis da região de Bofete/SP, obtiveram um erro padrão da estimativa para a produção volumétrica obtida pelo modelo de Clutter de 4,4\%. Nascimento (2010) encontrou um erro padrão da estimativa volumétrica pelo modelo de Clutter de 6,1\%, empregando dados do gênero Pinus, da região norte de Santa Catarina. Aplicando o modelo de Clutter para dados de eucaliptos da região sul da Bahia, Ferreira (2009) encontrou um erro padrão da estimativa de 5,7\% para a estimativa futura de produção volumétrica.

Por outro lado, a estratificação promoveu um $S_{y x} \%$ menor, de $5,86 \%$, ou seja, uma redução de $0,81 \%$. Acredita-se que se os dados de precipitação a serem utilizados na modelagem do crescimento e da produção provierem de estações meteorológicas locais, próximas à área de interesse, será possível obter 
melhores estimativas para a prognose da produção, ou mesmo se os dados de precipitação forem provenientes de isoietas em escala compatível com a área-foco da pesquisa.

\section{CONCLUSÕES}

- A precipitação média apresentou influências no crescimento médio em altura das árvores dominantes em função da idade, comprovada pelo teste proposto por Dette e Neumeyer (2001).

- O modelo de produção de Clutter foi adequado na obtenção das estimativas de volume e área basal, fornecendo valores coerentes do ponto de vista estatístico e biológico, tanto para os dados sem estratificação quanto para os dados estratificados por classes de precipitação pluviométrica

- A modelagem desenvolvida por classe de precipitação gerou resultados similares àqueles obtidos pela modelagem sem estratificação, não diferindo estatisticamente pelo teste $\mathrm{F}$ de Graybill, sendo consideradas adequadas para estimar a produção para povoamentos clonais de eucalipto.

- O modelo de projeção proposto por classes de precipitação poderá gerar estimativas mais acuradas se alimentado com dados de precipitação advindos de estações locais.

\section{REFERÊNCIAS}

ASSMANN, E. Waldertragskunde. Muenchen: BLV Verlagsgessellschaft, 1961. 435 p.

CAMPOS, J. C. C.; LEITE, H. G. Mensuração Florestal: perguntas e respostas. 4. ed. atual. ampl. Viçosa: Editora UFV, 2013. 605 p.

CAMPOS, J. C. C.; ROSAS, M. P.; LEITE, H. G. Comparação de alternativas de determinação da idade técnica de corte em plantações de eucalipto. Revista Árvore, Viçosa: v. 20, n. 1, p. 37 - 49, 1996.

CLUTTER, J. L. Compatible growth and yield models for loblolly pine. Forest Science, v. 9, n. 3, p. 354 - 371, 1963.

CluTter, J. L.; FORTSON, J. C.; PIENAAR, L. V.; BRISTER, G. H.; BAILEY, R. L. Timber management: a quantitative approach. New York: John Wiley \& Sons, 1983. 333 p.

CORONA, P.; SCOTTI, R.; TARCHIANI, N. Relationship between environmental factors and site index in Douglas-fir plantations in central Italy. Forest ecology and Management, v. 110, p. 195 - 207, 1998.

COTTRELl, A.; LUCCHETTI, R. Gretl: Gnu Regression, Econometrics and Time-series Library. Department of Economics, Wake Forest University, 2013. Disponível em: <http://gretl.sourceforge.net/>. Acesso em: 12 de junho de 2013.

COMPANHIA DE PESQUISA DE RECURSOS MINERAIS (CPRM). Mapa levantamento da geodiversidade. Projeto atlas pluviométrico do Brasil - Estado da Bahia. Isoietas anuais médias período 1977 a 2006: escala 1: 1.000.000, 2006. Disponível em: <http://www.cprm.gov.br/publique /media/Isoietas_Totais_Anuais_1977_2006_BA.pdf>. Acesso em: 2 de julho de 2012.

CRUZ, J. P. Crescimento e produção de Tectona grandis na região de Tangará da Serra - Mato Grosso. 41 p. Dissertação (Mestrado em Ciência Florestal) - Universidade Federal de Viçosa, Viçosa, 2005.

DAVIS, L. S.; JOHNSON, K. N. Forest management. 3. ed., New York: McGraw-Hill, 1987. 790 p.

DETTE, H.; NEUMEYER, N. Nonparametric analysis of covariance. Annals of Statistics, v. 29, n. 5, p. 1361 - 1400, 2001.

DIAS, A. N. Modelagem e avaliação econômica de plantações de eucalipto submetidas a desbastes. 70 p. Dissertação (Mestrado em Ciência Florestal) - Universidade Federal de Viçosa, Viçosa, 2000.

DIAS, A. N.; LEITE, H. G.; CAMPOS, J. C. C.; COUTO, L.; CARVALHO, A. F. Emprego de um modelo de crescimento e produção em povoamentos desbastados de eucalipto. Revista Árvore, Viçosa, v. 29, n. 5, p. 731 - 739, 2005.

DRAPER, N. R.; SMITH, H. Applied regression analysis. New York: John Wiley \& Sons, 1981. 709 p. 
FERREIRA, M. Z. Modelagem da influência de variáveis ambientais no crescimento e na produção de Eucalyptus sp. 101 p. Tese (Doutorado em Engenharia Florestal) - Universidade Federal de Lavras, Lavras, 2009.

FREIRE, J. P. A. Modelação do crescimento da árvore individual independente da distância para Pinus pinaster Aiton. em Portugal. 115 f. Dissertação (Mestrado em Silvicultura de Espécies de Crescimento Rápido) - Universidade Técnica de Lisboa, Universidade de Trás-os-Montes e Alto Douro, Lisboa, 2002.

GRAYBILL, F. A. Theory and application of the linear model. North Scituate, Massachusetts: Duxbury Press, 1976. 704 p.

HUNTER, I. R.; GIBSON, A. R. Predicting Pinus radiata site index from environmental variables. New Zealand Journal of Forestry Science, v. 14, p. 53 - 64, 1984.

KOEHLER, A. B. Modelagem biométrica e morfometria em povoamentos jovens de Araucaria angustifolia (Bert.) Ktze., em Tijucas do Sul, Estado do Paraná. 128 f. Tese (Doutorado em Engenharia Florestal) - Universidade Federal do Paraná, Curitiba, 2009.

MAESTRI, R. Modelo de crescimento e produção para povoamentos clonais de Eucalyptus grandis considerando variáveis ambientais. 143 f. Tese (Doutorado em Engenharia Florestal) - Universidade Federal do Paraná, Curitiba, 2003.

MIRANDA, R. O. V. Modelagem em nível de povoamento para Eucalyptus sp. com estratificação em classes de precipitação pluviométrica. 78 f. Dissertação (Mestrado em Ciências Florestais) Universidade Estadual do Centro-Oeste, Irati, 2012.

NASCIMENTO, F. A. F. Modelagem biométrica e planejamento florestal otimizado utilizando a meta-heurística enxame de partículas. 99 f. Dissertação (Mestrado em Ciências Florestais) Universidade Estadual do Centro-Oeste, Irati, 2010.

NOVAES, D. M. Modelagem de crescimento e produção de povoamentos de Tectona grandis L. f. a partir de análise do tronco. UFV: Viçosa, 2009. 42 f. Dissertação (Mestrado em Ciência Florestal) Universidade Federal de Viçosa, Viçosa, 2009.

OLIVEIRA, J. C. Modelagem de crescimento e produção de povoamentos de eucalipto empregando mínimos quadrados generalizados. UFV: Viçosa, 2012. 75 f. Dissertação (Mestrado em Ciência Florestal) - Universidade Federal de Viçosa, Viçosa, 2012.

R DEVELOPMENT CORE TEAM. R: A language and environment for statistical computing. $R$ Foundation for Statistical Computing, Vienna, Austria. ISBN 3-900051-07-0. Disponível em <http://www.r-project.org/>. Acesso em: 22 de setembro de 2012.

SANTANA, C.; MELlO, A. A.; EISFELD, R. L.; SANQUETTA, C. R. Sistema de equações para simulação do crescimento e da produção em povoamentos de Eucalyptus grandis Hill ex Maiden. sem desbaste baseado no modelo de Clutter. Revista Ambiência, Guarapuava, PR, v. 1, n. 2, p. 239 - 256, 2005.

SCHNEIDER, P. R.; SCHNEIDER, P. S. P.; SOUZA, C. A. M. Análise de regressão aplicada à engenharia florestal. FACOS-UFSM: Santa Maria, 2009. 294 p.

SCOLFORO, J. R. S. Biometria florestal: modelos de crescimento e produção florestal. Lavras: FAEPEUFLA, 2006. 393 p.

SOARES, T. S.; LEITE, H. G.; VALE, A. B.; SOARES, C. P. B.; SILVA, G. F. Avaliação de um modelo de passo invariante na predição da estrutura de um povoamento de Eucalyptus sp. Revista Árvore, Viçosa, v. 31, n. 2, p. 275 - 283. 2007.

SOUZA, M. J. H.; RIBEIRO, A.; LEITE, H. G.; LEITE, F. P.; MINUZZI, R. B. Disponibilidade hídrica do solo e produtividade do eucalipto em três regiões da bacia do rio Doce. Revista Árvore, Viçosa, v. 30, n. 3, p. $399-410,2006$. 
TEMPS, M. Adição da precipitação pluviométrica na modelagem do crescimento e da produção florestal em povoamentos não desbastados de Pinus taeda L. 83 f. Dissertação (Mestrado em Engenharia Florestal) - Universidade Federal do Paraná, Curitiba, 2005.

WANG, X. F. fANCOVA: Nonparametric Analysis of Covariance. R package version 0.5-1, 2010. Disponível em: <http://CRAN.R-project.org/package=fANCOVA>. Acesso em: 22 de setembro de 2012. 